\title{
IMPLANTAÇÃO DE UM OTIMIZADOR EM TEMPO REAL (RTO) NO CONVERSOR DE UMA UNIDADE DE CRAQUEAMENTO CATALÍTICO
}

\author{
Valmir J. Camolesi* \\ camolesiepetrobras.com.br
}

\author{
Lincoln F. L. Moro ${ }^{\dagger}$ \\ lmorodpetrobras.com.br
}

\author{
Antônio C. Zanin ${ }^{\dagger}$ \\ zanin@petrobras.com.br
}

*Petrobras - Refinaria Henrique Lage, São José dos Campos (SP)

${ }^{\dagger}$ Petrobras - Abastecimento/Refino, Rio de Janeiro (RJ)

\section{RESUMO}

Este artigo descreve a implementação de um otimizador em tempo real (RTO) no conversor da unidade de craqueamento catalítico em leito fluidizado (FCC) da Refinaria Henrique Lage da Petrobras. É utilizada uma estratégia de otimização em tempo real, na qual os valores ótimos das variáveis manipuladas/controladas calculados pelo otimizador são implementados através do algoritmo de programação quadrática (PQ) do controlador multivariável. O ciclo de otimização consiste nas seguintes etapas executadas de forma seqüencial: (1) detecção do estado estacionário do processo, (2) ajuste dos parâmetros do modelo, (3) solução do problema de otimização, o qual corresponde à máxima lucratividade da unidade dentro de uma região operacional limitada pelas restrições do processo e (4) integração com o controlador multivariável. O otimizador é constituído pelo software FCCSIM, licenciado pela empresa KBC, inserido no SICON (plataforma da Petrobras para implementação de controle avançado) para ser executado em tempo real. A estimativa dos benefícios econômicos é de US\$ 1,6 milhões por ano, obtidos através de aumento no rendimento de produtos mais valiosos e redução no consumo de energia.

PALAVRAS-CHAVE: Otimização em Tempo Real, Otimiza-

\footnotetext{
Artigo submetido em 03/05/2007

1a. Revisão em 18/02/08

2a. Revisão em 28/02/08

Aceito sob recomendação do Editor Associado Prof. José Roberto Castilho Piqueira
}

ção de FCC, Controle Avançado, RTO.

\begin{abstract}
This paper describes the implementation of a real time optimization (RTO) in a fluid catalytic cracking (FCC) converter of Henrique Lage Petrobras' refinery. It is used an RTO strategy in which the manipulated/controlled targets calculated by the optimizer are implemented by the multivariable predictive controller, via quadratic programming (QP). The optimization cycle consists on the following steps, executed in sequence: (1) steady state detection of process, (2) model parameter updating, (3) solution of optimization problem, that corresponds to the maximum profitability inside the operating region limited by the process constraints and (4) integration with the multivariable controller. The optimizer is composed of FCCSIM software, licensed by KBC Company, integrated with SICON (Petrobras' software for advanced control implementation) for on line execution. The economic benefit estimation is US\$1.6 million per year, due to increasing yields of more valuable products and energy saving.
\end{abstract}

KEYWORDS: Real Time Optimization, FCC Optimization, Advanced Control, RTO.

\section{INTRODUÇÃO}

A globalização tem conduzido à necessidade de otimização da operação de plantas químicas. Quanto mais competitivo 
é um mercado específico, mais sofisticadas devem ser as estratégias de controle e otimização da planta industrial relacionada ao mesmo. Este é o cenário típico de uma refinaria de petróleo, onde pequenas melhorias na sua operação podem resultar em grandes benefícios econômicos.

Segundo Cutler e Perry (1983), a otimização em tempo real da operação de uma unidade de processo é uma tarefa difícil e complexa, porém seus benefícios são relevantes, podendo incrementar, em conjunto com o controle avançado, de 6 a $10 \%$ a lucratividade da referida unidade. Carlberg et al. (1998) enfatizam a necessidade de uma visão global do negócio e a escolha apropriada das restrições do processo para o sucesso de um projeto de otimização em tempo real. Os benefícios da otimização dependem dos seguintes fatores (White, 1997b):

- disponibilidade de mercado para aumento da capacidade da unidade;

- diferenças de preços entre os produtos;

- magnitude do consumo específico de energia;

- número de variáveis manipuladas e restrições;

- precisão do modelo do processo.

A unidade de craqueamento catalítico em leito fluidizado (FCC) é utilizada para converter hidrocarbonetos de elevados pesos moleculares em produtos de maior valor econômico, como gasolina e gás liquefeito do petróleo (GLP). Para Ellis et al. (1998) a unidade de craqueamento catalítico é a candidata ideal para a implementação de otimização em tempo real, uma vez que a mesma é economicamente importante e apresenta interações complexas entre suas variáveis devido à sua acentuada não linearidade.

A melhor estratégia para otimizar uma unidade de FCC é ainda um problema desafiador. Diversas publicações tratam da modelagem, controle e otimização de unidades de craqueamento catalítico, dentre as quais estão: Grosdidier et al., 1993; McFarlane et al., 1993; Moro e Odloak, 1995; Arbel et al., 1995, McCormick et al., 1996; Chitnis e Corripio, 1998; Zanin, 2001.

O objetivo deste trabalho consiste na apresentação de uma estrutura de otimização em tempo real e na descrição da estratégia de otimização implementada no conversor de FCC da unidade U-220. O otimizador consiste no software FCCSIM licenciado pela empresa $\mathrm{KBC}$, o qual foi inserido no SICON (Moro, 1997) que é a plataforma da Petrobras para a implementação de aplicativos "on-line", tais como controle multivariável e inferências.

Este trabalho está organizado em cinco partes. Na seção 2 é apresentada uma estrutura de otimização em tempo real, a qual é a utilizada nas aplicações implementadas pelas empresas que comercializam este software. A seção 3 contém a definição do problema de otimização. São analisadas as variáveis manipuladas e as restrições do conversor, no qual é implementada a otimização. A seção 4 apresenta a formulação matemática do otimizador e do controlador multivariável ao qual a mesma está integrada. Finalmente a seção 5 descreve os aspectos de implementação do otimizador e apresenta os seus benefícios.

\section{ESTRUTURA DE OTIMIZAÇÃO EM TEMPO REAL}

A otimização de um sistema consiste na determinação de suas variáveis independentes, através de um modelo matemático do mesmo e um algoritmo de programação matemática, com a finalidade de maximizar ou minimizar uma função objetivo sem violar as restrições do referido sistema. A otimização é em tempo real quando a solução deste problema, dita ótima, é obtida periodicamente e implementada de forma automática.

Para o sistema analisado neste trabalho, o modelo é constituído de um conjunto de equações não lineares que buscam representar com fidelidade o seu estado estacionário. A função objetivo consiste em um modelo econômico que reflete a sua lucratividade. As restrições estão associadas aos limites dos seus equipamentos, especificações dos seus produtos e limitações impostas pelo mercado e meio ambiente. As variáveis independentes são constituídas pela maioria das manipuladas do respectivo controle avançado e algumas restrições do estado estacionário do sistema.

A otimização faz a conexão entre as tarefas de planejamento e programação da produção e as executadas pelo controlador multivariável. A figura 2.1 (Rotava e Zanin, 2005) ilustra a estrutura tradicional para a otimização de um sistema de múltiplas unidades, com seus componentes inter-relacionados.

Como fatores importantes na otimização em tempo real de um processo contínuo estão os seguintes itens:

a) Modelagem do sistema em estado estacionário

O modelo matemático deve representar o sistema em uma ampla faixa de operação e com elevado grau de precisão, de forma que, quando utilizado pelo otimizador, não sejam violadas as restrições do processo e a sua lucratividade potencial seja obtida.

\section{b) Caracterização da carga}

É necessário fornecer ao modelo do processo as propriedades da carga. Assim, a otimização em tempo real envolve a detecção da qualidade da carga que pode ser obtida através 


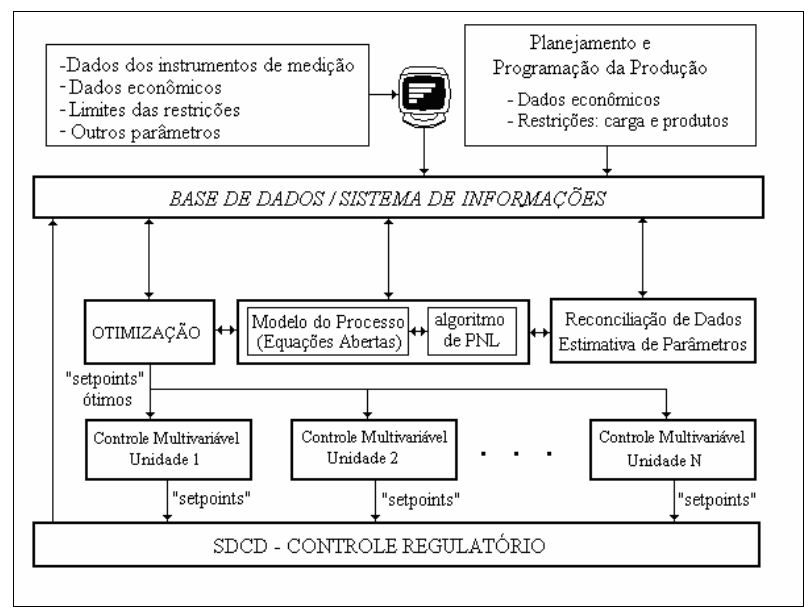

Figura 2.1 Estrutura tradicional para otimização em tempo real

de inferências ou analisadores em linha.

\section{c) Formulação da função objetivo}

Geralmente a função objetivo a ser maximizada é a lucratividade definida como o balanço econômico da unidade. De acordo com Friedman (1995), para que a otimização de uma unidade isolada forneça uma solução correta é necessário especificar corretamente o preço de cada produto, o qual depende da sua qualidade e quantidade produzida e em estoque, da programação da produção e planejamento futuro da refinaria. Segundo o referido autor, embora ainda não exista uma metodologia consolidada para a determinação dos preços de produtos intermediários, os mesmos podem ser fornecidos, através de valores econômicos marginais da programação linear (PL), pelo planejamento e programação de produção que contemplam um modelo simplificado abrangendo todas as unidades da refinaria.

\section{d) Algoritmo de detecção do estado estacionário}

A reconciliação de dados e a estimativa de parâmetros são efetuadas com a unidade no estado estacionário. Testes estatísticos, utilizando dados históricos recentes, são usados para a detecção do mesmo. Por exemplo, o estado estacionário do conversor de uma unidade de craqueamento catalítico poderia ser detectado através dos desvios padrões das temperaturas de reação e regeneração do catalisador, os quais seriam calculados a cada período de amostragem, utilizando um horizonte móvel correspondente à dinâmica do processo.

\section{e) Estimativa de parâmetros}

A natureza dos modelos rigorosos permite o ajuste de seus parâmetros para compensar perturbações não medidas e mudanças no desempenho do processo provocadas por fatores como desativação de catalisadores, incrustação em trocado- res de calor e redução de eficiência de fornos e colunas de destilação. O ajuste dos parâmetros do modelo corresponde à correção do mesmo através da ação de retroalimentação do processo.

\section{f) Algoritmo de otimização}

É utilizado um algoritmo de programação não linear (PNL) para o cálculo da solução ótima do problema de otimização, a qual corresponde à máxima lucratividade da unidade dentro de uma região operacional limitada pelas restrições do processo. Segundo White (1997a), o algoritmo de PNL normalmente utilizado em aplicações industriais é o SQP (Successive Quadratic Programming).

g) Integração do otimizador ao controlador multivariável

O controlador multivariável tem a função de implementar (via SDCD - Sistema Digital de Controle Distribuído) a solução fornecida pelo algoritmo de otimização e manter a unidade dentro de seus limites operacionais. O otimizador é executado de forma sincronizada com o controle avançado. A solução ótima normalmente é enviada ao controlador multivariável na forma de "setpoints" ideais para o estado estacionário de suas variáveis manipuladas/controladas.

\section{DEFINIÇÃO DO PROBLEMA DE OTIMI- ZAÇÃO}

\subsection{Descrição do processo}

O conversor da U-220, modelo F da Kellog, é ilustrado pela figura 3.1. A unidade é alimentada por duas correntes: gasóleo, proveniente de tanque, e óleo desasfaltado, o qual vem diretamente da unidade de desasfaltação a propano e contém um alto teor de hidrocarbonetos pesados. Estas correntes se juntam e a mistura, depois de aquecida no forno, é enviada para o "riser", onde ocorrem as reações de craqueamento.

No "riser", devido ao contato com o catalisador quente, a carga é rapidamente vaporizada e iniciam-se as reações de craqueamento, as quais continuam ao longo do "riser", onde o tempo de residência é de poucos segundos após a mistura da carga e catalisador. Os produtos da reação e o catalisador deixam o "riser" e entram primeiramente em um pseudociclone e em seguida em um conjunto de ciclones, onde o catalisador é separado da fase gasosa que contém os hidrocarbonetos. Estes são direcionados para a fracionadora principal.

Durante as reações de craqueamento ocorre a deposição de coque na superfície do catalisador, reduzindo drasticamente a sua atividade. A geração de energia para as reações endotérmicas de craqueamento e a reativação do catalisador são efetuadas, pela combustão do coque depositado no mesmo, 


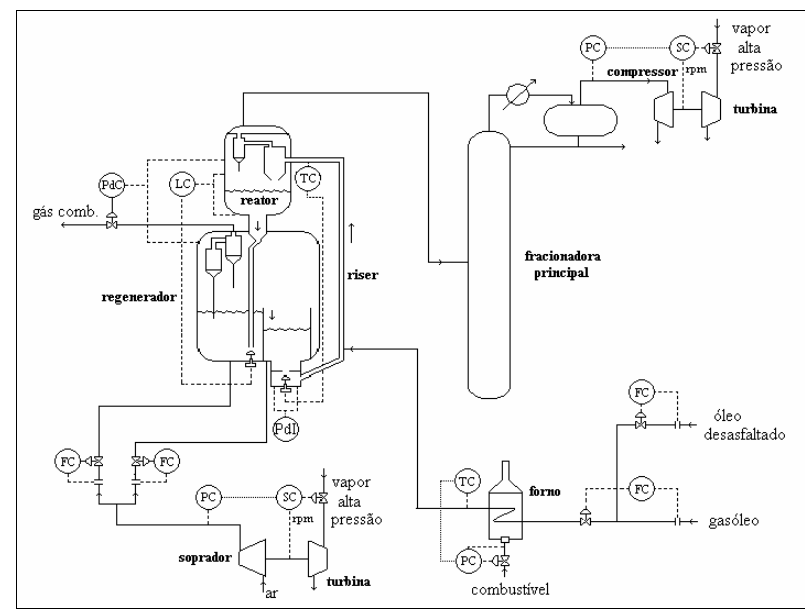

Figura 3.1 Esquema do conversor da U-220

no equipamento denominado regenerador, o qual é dividido em duas partes principais denominadas $1^{\circ}$ e $2^{\circ}$ estágios de regeneração. Cada um dos estágios é composto por uma fase densa formada por um leito fluidizado de catalisador e, sobre a mesma, uma fase diluída composta pelos gases de combustão e catalisador arrastado da fase densa. Sobre as fases diluídas do $1^{\circ}$ e $2^{\circ}$ estágios é formada a fase diluída geral. Como a combustão é parcial, os gases de combustão desta região são direcionados para a caldeira de $\mathrm{CO}$, onde pela conversão total do $\mathrm{CO}$ em $\mathrm{CO}_{2}$, ocorre geração de vapor de alta pressão.

O catalisador gasto, antes de ser conduzido ao regenerador, é mantido fluidizado no fundo do reator através da injeção de vapor de retificação, o qual remove os hidrocarbonetos adsorvidos no catalisador. Através de um controlador de nível, o catalisador gasto é depositado na fase densa do $1^{\circ}$ estágio, onde se inicia o processo de combustão. Posteriormente, o mesmo transborda sobre um vertedouro para o $2^{\circ}$ estágio, no qual é concluído o processo de regeneração. $\mathrm{O}$ ar necessário para o processo de queima do coque é fornecido pelo soprador, sendo constante a relação entre as vazões para os dois estágios de regeneração.

As principais malhas do controle regulatório do conversor são:

- Vazão de ar e temperatura do "riser": para a realização dos balanços energético e de carbono. Estes consistem na combustão de todo o coque gerado e na absorção desse calor pelas reações endotérmicas;

- Pressão da sucção dos compressores e diferencial de pressão entre o regenerador e o reator: atuam no balanço das pressões do sistema, determinando as vazões máximas de catalisador regenerado e ar.

\subsection{Software de otimização e modelo es- tático do conversor}

O software de otimização é o FCCSIM, o qual é constituído basicamente por um modelo estacionário do conversor (KBC Profimatics ${ }^{T M}$ ) e um algoritmo de programação não linear SLP (Successive Linear Programming). O modelo foi desenvolvido pela Profimatics e é comercializado pela KBC. A Petrobras possui licença do FCCSIM, o qual é utilizado nas suas refinarias para otimização "off-line" dos conversores de craqueamento catalítico.

O modelo KBC Profimatics ${ }^{T M}$ é rigoroso (baseado em balanço de massa, energia e cinéticas das reações de craqueamento/regeneração do catalisador).

Atualmente o FCCSIM é utilizado nas refinarias e é executado através de seus 3 modos:

- Calibração. Neste modo é ajustada uma série de parâmetros do modelo, tais como as constantes cinéticas das reações. Para tanto, são necessárias análises de laboratório da carga e todos os produtos e os dados operacionais do processo. A calibração é efetuada nos dias em que todas essas informações estão disponíveis;

- Predição. Uma vez ajustado o modelo e conhecidas as propriedades da carga, é possível determinar o rendimento e a qualidade de cada produto. Pode-se ainda determinar o efeito de variáveis de processo no desempenho da unidade;

- Otimização. Definida uma função objetivo (lucratividade, conversão ou rendimento de determinados produtos) e relacionadas as restrições do processo, são determinadas as correspondentes condições operacionais ótimas.

Informações detalhadas sobre o FCCSIM podem ser obtidas com a empresa KBC (www.kbcat.com).

\subsection{Variáveis e função objetivo do pro- blema de otimização do conversor}

A operação do conversor é complexa, devido à sua forte não linearidade e às elevadas interações entre suas variáveis. Existem várias restrições que não podem ser violadas. $\mathrm{O}$ maior número de variáveis manipuladas em relação às restrições ativas gera graus de liberdade para otimizar o processo.

Embora o FCCSIM tenha possibilidade de manipular até 18 variáveis independentes ou manipuladas, as selecionadas no conversor da U-220 são as seguintes: 
- temperatura da reação;

- temperatura da fase densa do $2^{\circ}$ estágio do regenerador;

- diferencial de pressão regenerador/reator;

- pressão de sucção do compressor;

- temperatura da carga combinada para o "riser".

Apesar da vazão e composição (razão entre gasóleo e óleo desasfaltado) da carga serem variáveis com grande sensibilidade na lucratividade da unidade, as mesmas não são manipuladas pelo otimizador, pois são determinadas pela programação da produção em função da otimização global da refinaria.

Além das restrições inseridas no controlador multivariável do conversor, foram também adicionadas a octanagem MON ("Motor Octane Number" - ASTM, 2007) da nafta craqueada e o teor de coque no catalisador regenerado.

A função objetivo a ser maximizada é a lucratividade definida como:

$$
\begin{aligned}
& \text { LUCRATIVIDADE }= \\
& \sum(\text { Vazão de Produto } \times \text { Preço do Produto }) \\
& -\sum(\text { Vazão da Carga } \times \text { Custo da Carga }) \\
& \quad-\text { Reposição } \times \text { Custo do Catalisador }) \\
& + \text { (Produção de Energia }- \text { Consumo de Energia }) \times
\end{aligned}
$$$$
\text { Preço da Energia }
$$

Os parâmetros econômicos (preços dos produtos e custos da energia e matéria prima) estão configurados na base de dados de processo $\mathrm{PI}^{T M}$ e são atualizados periodicamente.

\subsection{Variáveis e função objetivo do pro- blema de ajuste de parâmetros}

No ajuste de parâmetros ou calibração do modelo, as variáveis independentes ou manipualdas são os parâmetros do modelo do processo.

A função objetivo (FO), a ser minimizada no problema de ajuste de parâmetros, é definida como:

$$
F O=\left\|W_{V}\left(V^{p}-V\right)\right\|_{2}^{2}
$$

onde $\mathrm{V}$ é o vetor das variáveis operacionais da planta e dados de laboratório dos produtos; $\mathrm{V}^{p}$ é o vetor das mesmas variá- veis de $\mathrm{V}$, porém determinadas pelo modelo e $\mathrm{W}_{v}$ é a matriz dos pesos das variáveis na função objetivo.

Assim, no modo de ajuste, o algoritmo modifica os valores dos parâmetros procurando fazer com que os valores das variáveis calculadas pelo modelo $\left(\mathrm{V}^{p}\right)$ sejam os mais próximos quanto possível dos valores reais (V).

O modo calibração do FCCSIM ajusta mais de 400 parâmetros do modelo.

\subsection{Propriedades da carga}

A carga é caracterizada através das seguintes propriedades: densidade, ponto de anilina, teor de nitrogênio básico, teor de enxofre, curva de destilação ASTM, resíduo de carbono, teores de metais (ferro, níquel, vanádio, sódio e cobre) e viscosidade. Atualmente são fornecidos dados de análises de laboratório (disponíveis no $\mathrm{PI}^{T M}$ ) para as duas correntes que compõem a carga da unidade (gasóleo e óleo desasfaltado, conforme ilustração da figura 3.1) e as propriedas da mistura das mesmas são calculadas através do modelo do processo. Está sendo estudada a possibilidade de instalação de analisador em linha para cada corrente de carga.

\section{FORMULAÇÃO MATEMÁTICA}

A figura 4.1 (Rotava e Zanin, 2005) ilustra a otimização em três camadas, a qual é a estrutura clássica envolvendo o controle multivariável e a otimização em tempo real, sendo utilizada na maioria das aplicações industriais. A camada superior corresponde ao otimizador não linear (RTO) com modelo estático rigoroso do processo, a intermediária ao otimizador linear do controlador multivariável e a inferior ao controlador. A otimização linear e o controle são resolvidos seqüencialmente e na mesma freqüência. A camada superior é executada em uma freqüência relativamente baixa, cujo valor depende da complexidade do modelo do processo.

\subsection{Otimizador não linear}

O otimizador não linear é executado em uma freqüência menor que a do controlador multivariável (em torno de 2 horas no caso do conversor). As funções do mesmo são:

- maximizar a função objetivo econômico;

- manter as variáveis de processo, no estado estacionário, dentro dos seus limites operacionais;

- disponibilizar os valores ótimos das variáveis manipuladas e/ou controladas para a camada de otimização linear do controle multivariável. 


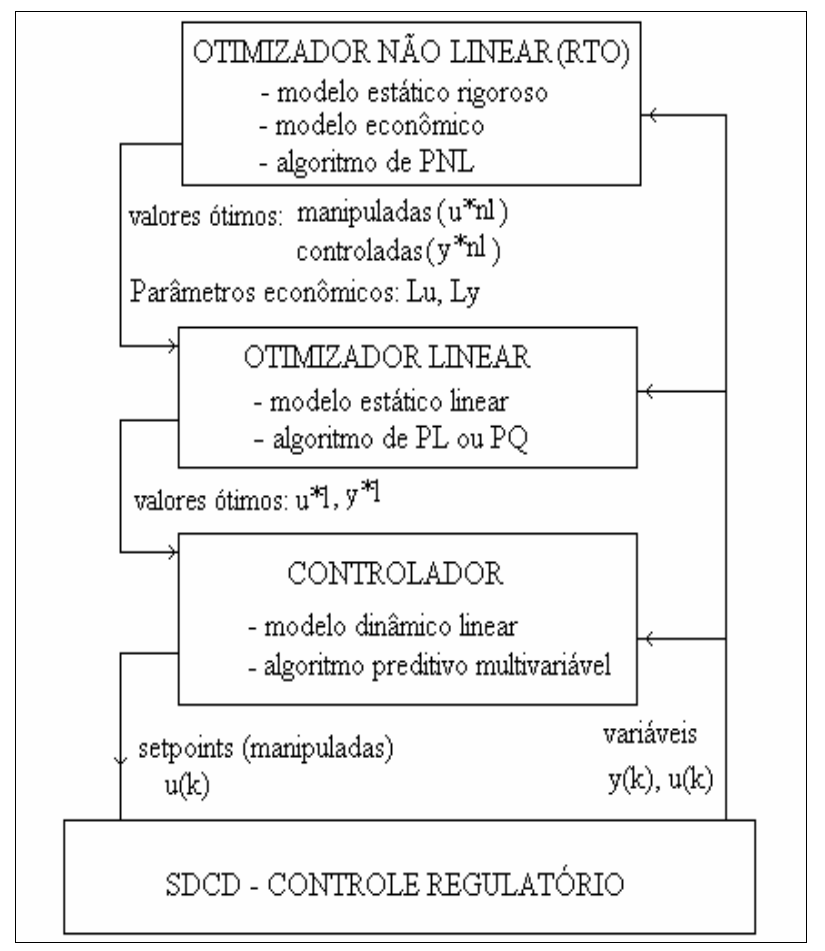

Figura 4.1 Estrutura clássica de otimização em tempo real

O problema de otimização é definido como:

$$
\max _{x_{\mathrm{S}}, \mathrm{u}_{\mathrm{S}}} \text { feco }
$$

Sujeito às restrições:

$$
\begin{gathered}
h_{p}\left(\mathrm{x}_{\mathrm{S}}, \mathrm{u}_{\mathrm{S}}, \mathrm{d}_{\mathrm{S}}\right)=0 \\
h_{e}\left(\mathrm{f}_{\mathrm{eco}}, x_{s}, \mathrm{u}_{\mathrm{s}}, \mathrm{d}_{\mathrm{S}}\right)=0 \\
u_{s}^{\text {inf }} \leq u_{s} \leq u_{s}^{\text {sup }} \\
x_{s}^{\text {inf }} \leq x_{s} \leq x_{s}^{\text {sup }}
\end{gathered}
$$

onde: $\mathrm{d}_{s}$ é o vetor das perturbações no estado estacionário; $\mathrm{f}_{e c o}$ é a função objetivo econômico (equação 3.1); $\mathrm{h}_{e}$ são as restrições do modelo econômico; $\mathrm{h}_{p}$ são as restrições do modelo não linear do conversor; $\mathrm{u}_{s}$ é o vetor das variáveis manipuladas no estado estacionário e $\mathrm{x}_{s}$ é o vetor das restrições no estado estacionário.

\subsection{Otimizador linear}

A função do otimizador linear consiste na determinação dos valores ótimos das variáveis manipuladas e/ou controladas para o controlador multivariável. Como este algoritmo é executado na mesma freqüência que a do controlador multivariável (1 minuto), a função do mesmo consiste na:

a) Otimização linear do processo quando o RTO estiver desligado.

O problema é definido como:

$$
\min _{u s, z}-W_{e} u s+\left\|W_{s s}(u s-u a t)\right\|_{2}^{2}+\left\|W_{r} z\right\|_{2}^{2}
$$

Sujeito às restrições da equação (4.4) e:

$$
x_{s}^{\mathrm{inf}} \leq x_{p s}+z \leq x_{s}^{\mathrm{sup}}
$$

b) Implementação da solução do otimizador não linear, rejeitando as perturbações do processo.

O problema é definido como:

$\min _{u s, z}\left\|W_{n l}\left(u s-u^{* n l}\right)\right\|_{2}^{2}+\left\|W_{s s}(u s-u a t)\right\|_{2}^{2}+\left\|W_{r} z\right\|_{2}^{2}$

Sujeito às restrições das equações (4.4) e (4.6).

onde: $\mathrm{u}^{* n l}$ é o vetor dos valores ótimos das variáveis manipuladas determinados pelo otimizador através de um modelo não linear; $\mathrm{u}^{\text {at }}$ é o vetor das variáveis manipuladas no instante atual; $\mathrm{W}_{e}$ é a matriz dos pesos econômicos das variáveis manipuladas; $\mathrm{W}_{n l}$ é a matriz dos pesos para conduzir as variáveis manipuladas para os seus respectivos valores ótimos determinados pelo RTO; $\mathrm{W}_{r}$ é a matriz diagonal dos pesos das restrições; $\mathrm{W}_{s s}$ é a matriz diagonal dos fatores de supressão das variáveis manipuladas; $\mathbf{x}_{p s}$ é o vetor da predição linear das variáveis no estado estacionário e z é o vetor das variáveis de folga.

\subsection{Controlador multivariável}

As funções do controlador são:

- manter as variáveis de processo dentro dos seus limites operacionais;

- conduzir as variáveis manipuladas para os seus valores ótimos determinados pelo otimizador linear. 
Para o controlador tem-se um problema dinâmico:

$$
\begin{gathered}
\min _{\Delta u(j T) ; j=1, \ldots, n l} \sum_{i=1}^{n r}\left\|W_{d}\left(y_{p}(i T)-y_{l}\right)\right\|_{2}^{2}+ \\
\sum_{j=1}^{n l}\left\|W_{s d} \Delta u(j T)\right\|_{2}^{2}+ \\
\sum_{j=1}^{n l}\left\|W_{l}\left(u_{\mathrm{at}}+\sum_{\mathrm{k}=1}^{\mathrm{j}} \Delta u(k T)-u^{* 1}\right)\right\|_{2}^{2}
\end{gathered}
$$

Sujeito às restrições dinâmicas do processo.

onde: nl é o horizonte de controle; nr é o horizonte de predição; T é o período de amostragem ou de execução do algoritmo; $\Delta \mathrm{u}$ é o vetor das amplitudes das ações de controle; $\mathrm{u}^{* l}$ é o vetor dos valores ótimos das variáveis manipuladas determinados pelo otimizador linear; $\mathrm{W}_{l}$ é a matriz dos pesos para conduzir as variáveis manipuladas para os seus respectivos valores ótimos determinados pelo otimizador linear; $\mathrm{W}_{r}$ é a matriz diagonal dos pesos das variáveis controladas dinamicamente; $\mathrm{W}_{s d}$ é a matriz diagonal dos fatores de supressão das variáveis manipuladas; y $y_{l}$ é o vetor dos "setpoints" modificados das variáveis controladas dinamicamente e $\mathrm{y}_{p}$ é o vetor da predição linear das variáveis controladas dinamicamente.

As restrições dinâmicas da equação (4.8), bem como os cálculos de $\mathrm{x}_{p s}, \mathrm{y}_{l}$ e $\mathrm{y}_{p}$ estão relacionados em Zanin (2001).

\section{IMPLEMENTAÇÃO DO OTIMIZADOR}

O FCCSIM é utilizado pelos engenheiros de processo das refinarias para otimização "off-line" através da execução seqüencial do software nos seus 3 modos descritos no item 3.2 (calibração, predição e otimização). Isso exige que o engenheiro de processo execute um procedimento em uma planilha Excel para executar o FCCSIM e, no caso de implementação da solução, é também necessária a alteração de parâmetros e limites de variáveis na interface de engenharia do controle avançado.

O desafio em implantar o FCCSIM em tempo real ("on-line") consistia em executá-lo periodicamente durante as 24 horas do dia, analisar seus resultados e enviar os mesmos ao controle avançado para serem implementados no SDCD. Desta forma foi desenvolvido um programa denominado "gerenciador de RTO" para realizar a interface com o FCCSIM (enviar/receber informações) e executar o ciclo do otimizador.

\subsection{Ciclo de execução do otimizador}

O ciclo do algoritmo de otimização, executado em média uma vez a cada 2 horas, está ilustrado na figura 5.1, cujas etapas são:

a) Inicialização. Consiste na inicialização de variáveis, na leitura do arquivo dos parâmetros geométricos do conversor e busca dos índices das informações localizadas na base de dados do processo $\left(\mathrm{PI}^{T M}\right)$.

b) Leitura das variáveis. Nesta etapa são realizadas as leituras das variáveis de processo e efetuadas as validações e filtragem das mesmas. As variáveis são obtidas da base de dados do processo ( $\mathrm{PI}^{T M}$ ) que é atualizada através do SDCD. Também são obtidas informações para o RTO armazenadas no $\mathrm{PI}^{T M}$, como dados de laboratório, preços de produtos e custos das matérias primas.

c) Detecção do estado estacionário. É efetuada através de análise estatística de dados históricos de um conjunto de variáveis do conversor.

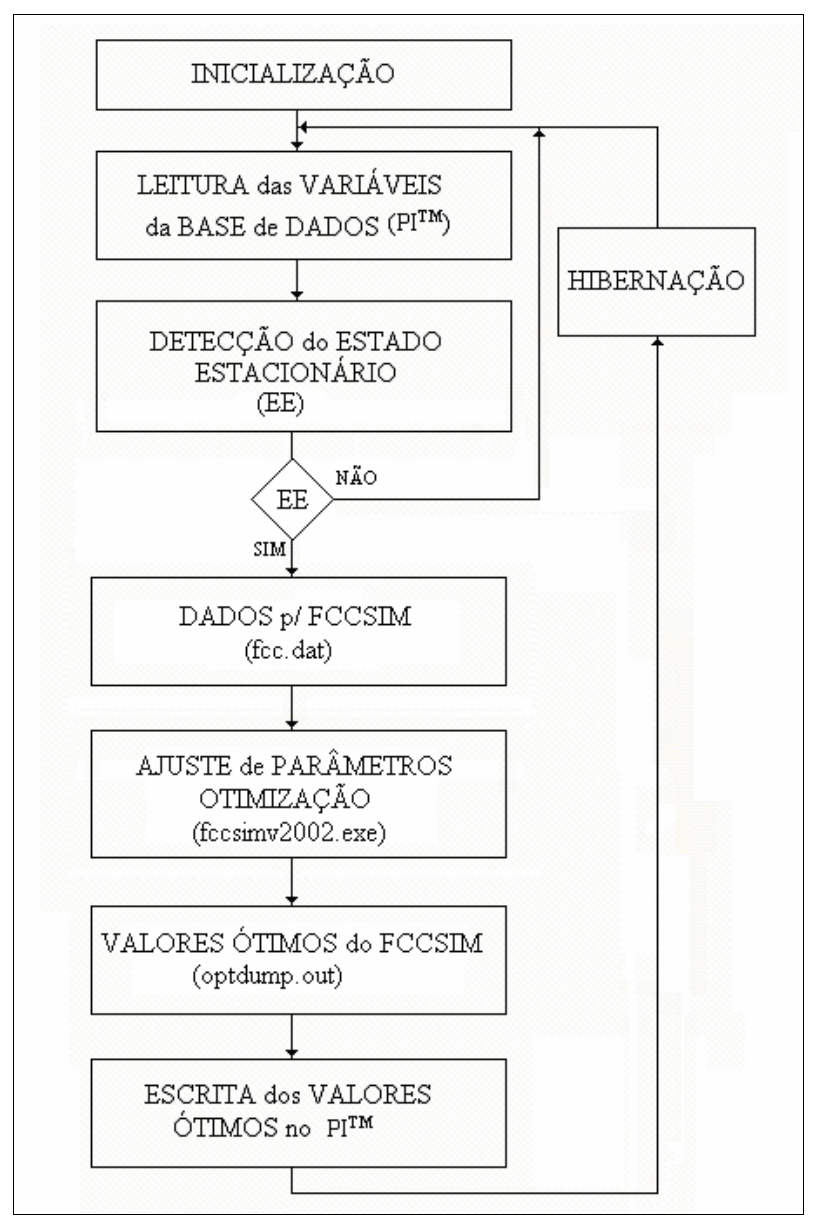

Figura 5.1 Ciclo de execução do otimizador 
d) Preparação dos dados para o otimizador (FCCSIM). Com a unidade em estado estacionário, é gerado o arquivo de dados "fcc.dat" para executar seqüencialmente o ajuste de parâmetro e a otimização. Essas informações são:

- dados de processo da unidade;

- parâmetros geométricos do conversor;

- análise de laboratório das cargas e produtos;

- análises do catalisador;

- preços de produtos e custos das cargas e de energia;

- variáveis independentes e seus respectivos limites;

- restrições e seus respectivos limites.

e) Ajuste de parâmetros e otimização. São executados de forma seqüencial. Os parâmetros atualizados são utilizados no modo otimização.

f) Leitura dos valores ótimos do FCCSIM. O arquivo "optdump.out"contém os resultados do otimizador:

- código de retorno referente à obtenção da solução ótima;

- função objetivo após o ajuste do modelo e após a otimização;

- valores ótimos das variáveis independentes;

- valores das restrições na solução otimizada.

g) Escrita dos valores ótimos no $\mathrm{PI}^{T M}$. Os mesmos são lidos desta base de dados pelo controlador multivariável.

\subsection{Interface de operação}

A Figure 5.2 ilustra a tela de operação do RTO, a qual está localizada no SDCD.

Na parte superior da tela estão:

ATIVAÇÃO: botão para ligar (LIGA) e desligar (DESLIGA) a aplicação.

STATUS: sinalização indicando se o otimizador está atuando (LIGADO) ou não (DESLIGADO) no processo. Também indica a disponibilidade de uma solução ótima relativamente recente (DISPONÍVEL), para manter o RTO ligado ou fornecer condição para ligá-lo.

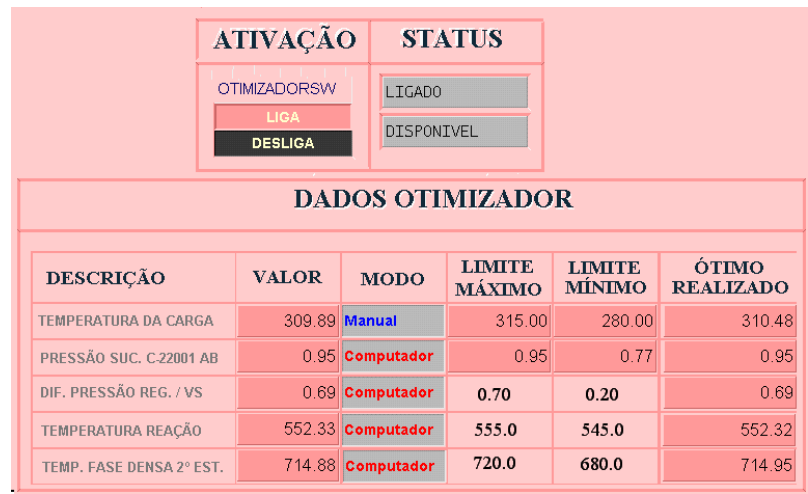

Figura 5.2 Interface do RTO com a operação

A parte central da tela contém as variáveis manipuladas pelo otimizador. São apresentadas as seguintes informações:

VALOR: valor de processo lido da planta.

MODO: modo do controlador ajustado pela operação.

LIMITE MÁXIMO e LIMITE MÍNIMO: mínimo e máximo das variáveis independentes, definidos pela engenharia de processo e operação e escritos no SDCD pelo gerenciador do otimizador. As variáveis que pertencem ao otimizador e controle avançado possuem os mesmos limites em ambas aplicações.

ÓTIMO REALIZADO: última solução do otimizador implementada pelo controle avançado.

O procedimento para ligar o otimizador consiste em:

1. Verificar se o otimizador está disponível: STATUS = DISPONÍVEL.

2. Transferir as variáveis manipuladas pelo otimizador para o modo computador (HOST).

3. Pressionar o botão LIGA. A partir de então o STATUS = LIGADO sinaliza que a solução do RTO está sendo implementada.

Conforme pode ser observado na figura 5.2, atualmente a temperatura da carga não é manipulada pelo RTO uma vez que o respectivo controlador opera em manual em virtude do forno da unidade operar com uma carga térmica bastante elevada.

\subsection{Resultados do Otimizador}

O RTO do conversor da U-220 vem operando em malha fechada desde maio de 2006. Medições preliminares estimam 


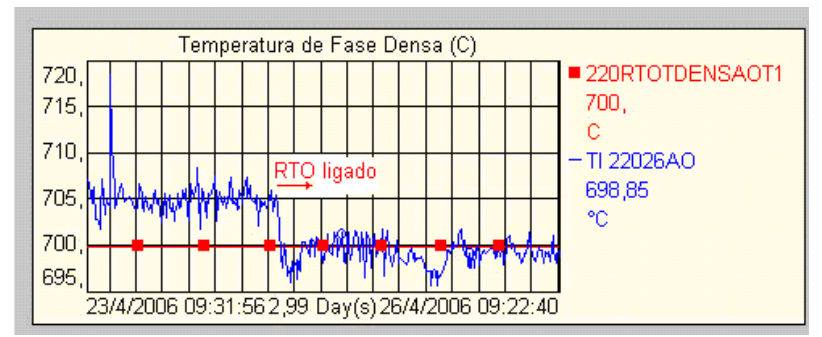

Figura 5.3a Temperatura da fase densa do $2^{\circ}$ estágio do regenerador

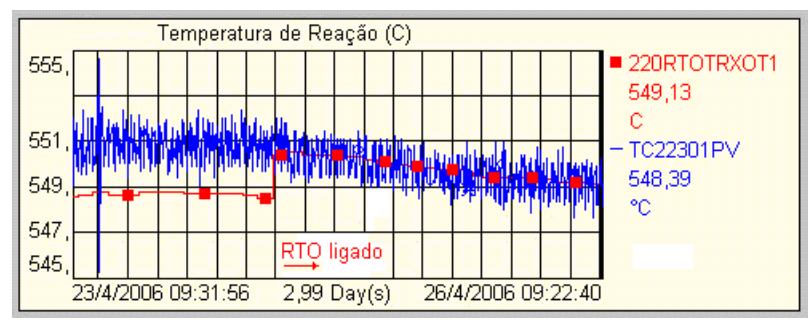

Figura 5.3b Temperatura da reação

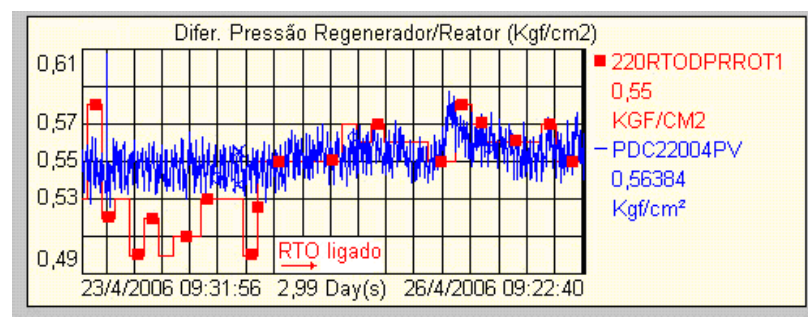

Figura 5.3c Diferencial de pressão regenerador/reator

um benefício anual em torno de US\$ 1.600.000,00. Este ganho é obtido com aumento no rendimento de produtos mais valiosos e redução no consumo de energia.

O valor acima está de acordo com a SOLOMON (1994) que estima o ganho econômico do RTO de uma unidade completa de craqueamento catalítico (vazão e composição da carga, conversor, fracionadora e sistema de recuperação de gases) em torno de US\$ 0,10 por barril de carga processada (US\$ 3.200.000,00 anual para a U-220).

As figuras 5.3a-d ilustram a atuação do RTO nas variáveis manipuladas efetivas do conversor da U-220. A curva sinalizada com retângulos corresponde aos valores ótimos calculados pelo otimizador e a outra, mais ruidosa, às medições das variáveis de processo.

Na figura 5.4 está representado o lucro potencial da otimização em tempo real, o qual é função das manipuladas disponíveis e suas respectivas faixa de atuação. Verifica-se que ao ser ligado o otimizador o lucro potencial é reduzido, indicando a realização do lucro através da implementação da solução ótima pelo controle avançado.

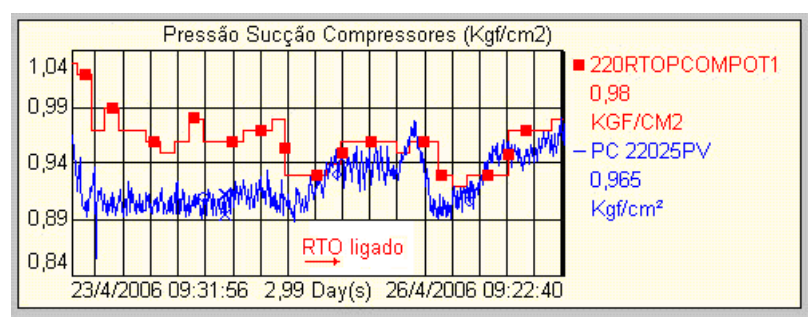

Figura 5.3d Pressão de sucção do compressor

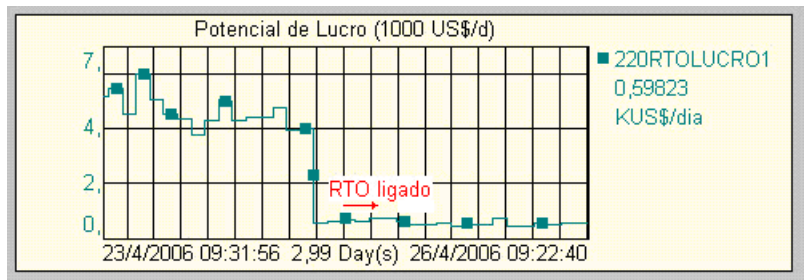

Figura 5.4 Lucro potencial do RTO

\section{CONCLUSÃO}

Essa aplicação de RTO foi pioneira nas refinarias da Petrobras. O sucesso obtido incentiva sua propagação às demais Unidades da Companhia.

A superação do desafio de otimizar resultados operacionais tem um significado especial para a Petrobras, pois além do grau de dificuldade inerente às aplicações pioneiras, o RTO ratifica a capacidade dos técnicos da companhia (REVAP, CENPES e AB-RE), tanto no aspecto de conhecimento como no da integração.

\section{REFERÊNCIAS}

Arbel, A.; Rinard, I. H. and Shinnar, R. (1995). Control strategies for fluid bed catalytic crackers: interaction of design and control. AIChE 1995 Spring National Meeting, paper 85f, Houston, March 19-23.

ASTM International (2007). Standard Test Method for Motor Octane Number of Spark-Ignition Engine Fuels, ASTM D2700.

Carlberg, N.; Coon, A. and Crowe, T. (1998). Computing Optimisation. Hydrocarbon Engineering, November, pp. 61-66,

Chitnis, U. K. and Corripio, A. B. (1998). On-line optimization of a Model IV catalytic cracking unit. ISA Transactions, Vol. 37, pp. 215-226.

Cutler, C. R. and Perry, R. T. (1983). Real-time optimization with multivariable control is required to maximizes profits. Computers and Chemical Engineering, Vol. 7, No. 5, pp. 663-667. 
Ellis, R. C.; Li, X. and Riggs J. B. (1998). Modeling and optimization of a Model IV fluidized catalytic cracking unit. AIChE Journal, Vol. 44, No. 9, pp. 2068-2079.

Friedman, Y. Z. (1995). What's wrong whit unit closed loop optimization? Hydrocarbon Processing, October, pp. 107-116.

Grosdidier, P.; Mason, A.; Aitolahti, A.; Heinonen, P. and Vanhamäki V. (1993). FCC unit reactor-regenerator control. Computers and Chemical Engineering, Vol. 17, No. 2, pp. 165-179.

McCormick, R.; Gagné, N.; Steven, S.; Laurier, G.; Brown, M. and Piette, R. (1996). Real-time optimization of fluid catalytic cracking and catalytic polymerization units. NPRA Computer Conference, National Petroleum Refiners Association, Atlanta, November 11-13, Proceedings.

McFarlane, R. C.; Reineman, R. C.; Bartee, J. F. and Georgakis, C. (1993). Dynamic simulator for a Model IV fluid catalytic cracking unit. Computers and Chemical Engineering, Vol. 17, No. 3, pp. 275-300.

Moro, L. F. L. and Odloak, D. (1995). Constrained multivariable control of fluid catalytic cracking converters. Journal of Process Control, Vol. 5, No. 1, pp. 29-39.

Moro, L. F. L. (1997). SICON - Uma solução cliente servidor para controle avançado. IV Congresso Petrobras de Informática e Telecomunicações - INFTEL, São Paulo.

Rotava, O. and Zanin, A. C. (2005). Multivariable control and real-time optimization - an industrial practical view. Hydrocarbon Processing, June, pp. 61-71.

SOLOMON (1994) Relatório de Controle Avançado, Otimização e Analisadores em Linha, Petrobras.

White, D. C. (1997a). The impact of new computer and communication technology on refinery operations. NPRA Annual Meeting, National Petroleum Refiners Association, paper AM-97-22, San Antonio, March 16-18, Proceedings.

White, D. C. (1997b). Online optimization: what, where and estimating ROI. Hydrocarbon Processing, June, pp. 4351.

Zanin A. C. (2001). Implementação industrial de um otimizador em tempo real. Tese (Doutorado) - Escola Politécnica, Universidade de São Paulo, São Paulo. 